\title{
Frequency-detuning dependent transient coaxial rf coupler kick in an $L$-band long-pulse high-gradient rf photogun
}

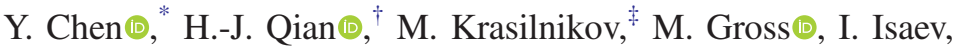 \\ G. Loisch๑, A. Oppelt $\odot$, and F. Stephan $\odot$ \\ Deutsches Elektronen-Synchrotron DESY, 15738 Zeuthen, Germany
}

(Received 12 October 2019; published 21 January 2020)

\begin{abstract}
A transverse kick resulting from the coaxial rf coupler in the L-band rf gun at the photoinjector test facility at DESY in Zeuthen (PITZ) has been systematically studied in simulations and further characterized in experiments. The used rf macro-pulse for the gun is typically $600 \mu \mathrm{s}$ long and applied to produce a train of up to 2700 electron bunches. The kick is transient and found to be dependent on the detuning of the resonance frequency of the gun cavity. The frequency detuning within the rf pulse results in a variation in the kick strength along the pulse. This leads to a downstream orbit and size change of individual bunches within the train. Using 3D rf field distributions, calculated at resonant and detuned frequencies of the cavity, particle tracking simulations are performed to evaluate the integral kick location and strength, simulate its transient behaviors with respect to gun launch phase and detuned frequency of the gun cavity, and thereby emulate its impacts onto the electron bunch. In the experiments, the temperature of the cooling water for the gun is tuned, allowing detailed characterization of the frequency detuning within the rf pulse and thus measurements of the kick which are of practical interest for machine operation. The resulting orbit and size change along a $240 \mu \mathrm{s}$ bunch train have been measured downstream at the injector exit for an rf gun peak power of about 5.7 MW. A measured focusing difference between head and tail of the bunch train is evaluated in terms of the gun solenoid current which provides more relevant information for overall tuning of the FELs. This is exemplarily given for both the under- and over-focusing case. The obtained results will be presented and discussed.
\end{abstract}

DOI: 10.1103/PhysRevAccelBeams.23.010101

\section{INTRODUCTION}

The European X-ray Free-Electron Laser (EuXFEL) provides excellent opportunities in pursuing excellence in frontier science and advanced interdisciplinary applications of coherent $\mathrm{x}$-ray radiation [1-4]. The photoelectron source in use at the EuXFEL is a photocathode of gun developed at the photoinjector test facility at DESY in Zeuthen (PITZ) [5,6]. The key component of the PITZ gun is a 1.6-cell $1.3 \mathrm{GHz}$ copper resonator. The gun can be operated with a high electric field gradient of about $60 \mathrm{MV} / \mathrm{m}$ on the cathode surface with long rf pulses of up to $650 \mu$ s at a repetition rate of $10 \mathrm{~Hz}$. This allows the production of a maximum number of 27000 bunches per second at the EuXFEL and results in a high peak rf power of about $6.5 \mathrm{MW}$ in the cavity. An average rf power of

\footnotetext{
*ye.lining.chen@desy.de

Present address: DESY, 22607 Hamburg.

thoujun.qian@desy.de

"mikhail.krasilnikov@desy.de
}

Published by the American Physical Society under the terms of the Creative Commons Attribution 4.0 International license. Further distribution of this work must maintain attribution to the author(s) and the published article's title, journal citation, and DOI. about $42 \mathrm{~kW}$ dissipates in the cavity with a length of about $20 \mathrm{~cm}$ and a radius of about $9 \mathrm{~cm}$. The rf power fed to the gun is supplied by a $10 \mathrm{MW}$ multibeam klystron. The power is coupled from the input waveguide via a door-knob transition into the rotationally symmetric coaxial coupler and the gun cavity, as shown in Fig. 1. Interested readers are referred to [5-7] for a detailed description of the gun and its supporting rf system.

The power coupler of a rf gun is a designed network for efficiently transferring power from a rf power source to the gun cavity $[9,10]$. The coupler design intrinsically integrates with complex technical requirements [11-16] and requires dedicated methods and techniques [17-19]. It is often that complementary devices need to be introduced in order to compensate the undesired effect(s) induced by the coupler $[15,16,20-26]$. In general, the power coupler introduces an asymmetry to the cylindrical symmetry of the fundamental electromagnetic mode of the gun cavity $[9,10]$. This distorts the rf field distribution of the fundamental mode. Due to the Lorentz force in the cavity, the distorted rf fields induce a time-dependent transverse deflection to the electrons within an accelerating bunch. The induced time-dependent kick, typically composed of multipole field components, causes transverse emittance growth and deteriorates beam qualities [25-30]. A brief 


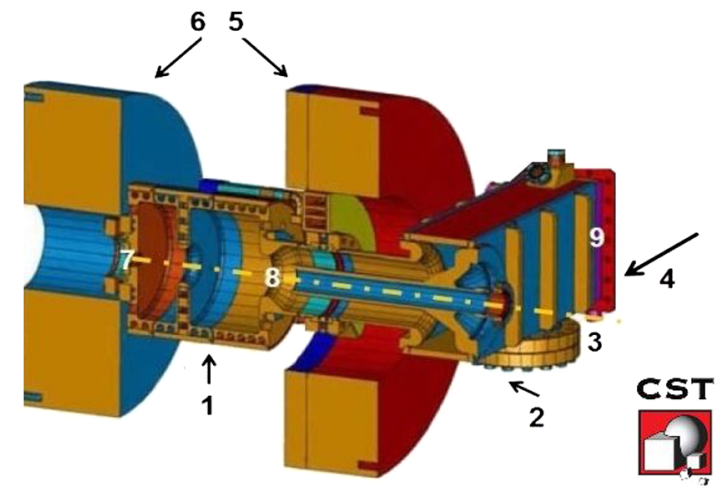

FIG. 1. Sketch of the PITZ gun in CST Microwave Studio [8]: 1-gun cavity, 2-door-knob transition, 3-cavity axis, 4-rf feeding direction, 5-main solenoid, 6-bucking solenoid, 7-cathode, 8-end of coaxial line, and 9-reference position of waveguide port for simulations. Note that, this sketch is rotated by 90 degrees compared to the computational model used for simulations.

review is given on relevant studies in the following. A waveguide dual-feed coupler design was applied to (the full cell of) the LCLS gun [20]. The existing coupler dipole kick was eliminated but a quadruple kick component remained. A racetrack shape was then adopted for the coupler cell to successfully damp the quadruple field [21]. A dual-feed racetrack coupling scheme was analyzed in detail for the LLNL gun [15]. For the LCLS-II FEL [22], the APEX VHF-gun developed at LBNL [31-33] is adopted as the electron source. The power coupling is realized through two diametrically opposed, magnetic loop couplers on the cathode back wall of the cavity [34]. At PAL, a four-hole design was proposed [23] for the rf gun to minimize the multipole field, however, the pulsed heating may cause a considerable issue. In [16], an asymmetric vacuum port design [24] and a four-port scheme were applied to a BNL-type rf gun built at Tsinghua University for the SXFEL project [35]. The multipole fields resulting from the rf coupling port were significantly reduced. At PITZ [5], a coaxial input coupler was designed to provide rotationally symmetric, on-axis rf input to the gun cavity $[11,12]$. The axial symmetry of the cavity thus stays undisturbed. Besides, detailed methods in [25] of minimizing the emittance growth due to coupler kicks in linear accelerators were investigated. For deflecting kick studies of a supposedly dipole symmetric waveguide coupler, simulations in [36] showed the complex nature of the superposed total field in the coupler near the beam axis and demonstrated a significant kick contribution from the traveling wave component which was beam loading dependent. In [26], analytic, numerical and experimental studies were systematically carried out for precise cancellation of the emittance growth due to coupled transverse dynamics in rf couplers.

Adoption of the coaxial input coupler for the PITZ gun, compared to the conventional input coupler aside the cavity for the TTF injector [29], has strongly suppressed the asymmetric electromagnetic modes inside the gun cavity. However, due to a single-side waveguide rf feed, the evanescent dipole mode ( $\mathrm{TE}_{11}$ mode) of the coaxial line is not avoidable to be excited at the door-knob transition (2 in Fig. 1). The dipole mode is not fully decayed till the end of the relatively short coaxial line and thus disturbs the cylindrical symmetry of the fundamental transverse electromagnetic mode. The induced rf field asymmetries create a transverse kick onto the electron bunch [37,38]. This occurs, more specifically, when the bunch is leaving the cavity through the inner conductor of the coaxial line (8 in Fig. 1).

In this paper, the gun coupler kick study is extended from the above-mentioned single-bunch effects studied in earlier works to bunch train effects, which are more relevant for the tuning of self-amplified spontaneous emission (SASE) FELs operated with long bunch trains. Both computational and experimental findings of the gun coupler rf kick will be presented. In Sec. II, a frequency domain field simulation is performed to clarify local rf field asymmetries by the end of the coaxial line (8 in Fig. 1). Using the obtained 3D rf field distribution, particle tracking simulations are conducted to identify the kick location in Sec. III and evaluate the kick strength in Sec. IV. The frequency-detuning dependency of the kick is analyzed in Sec. V with simulation results and measurement data shown in Secs. V B and V C, respectively. Measurements for clarifying the impacts of the kick onto bunch train operation are reported in Sec. VI. The measured downstream orbit and size change of individual bunches along a $240 \mu$ s train are presented in Sec. VI A and Sec. VI B, respectively.

\section{RF FIELD ASYMMETRIES}

The rf field in the gun is computed using the frequency domain solver in CST Microwave Studio (CST-MWS) [8].

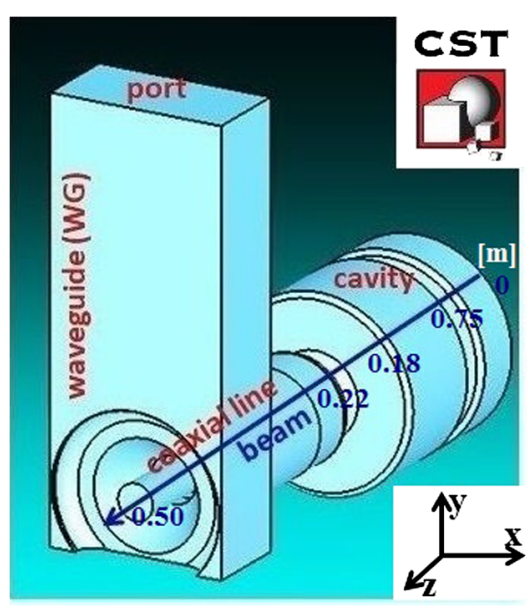

FIG. 2. A computational gun model in CST-MWS for frequency domain calculation. The inset numbers indicate the longitudinal positions along the cavity axis. 

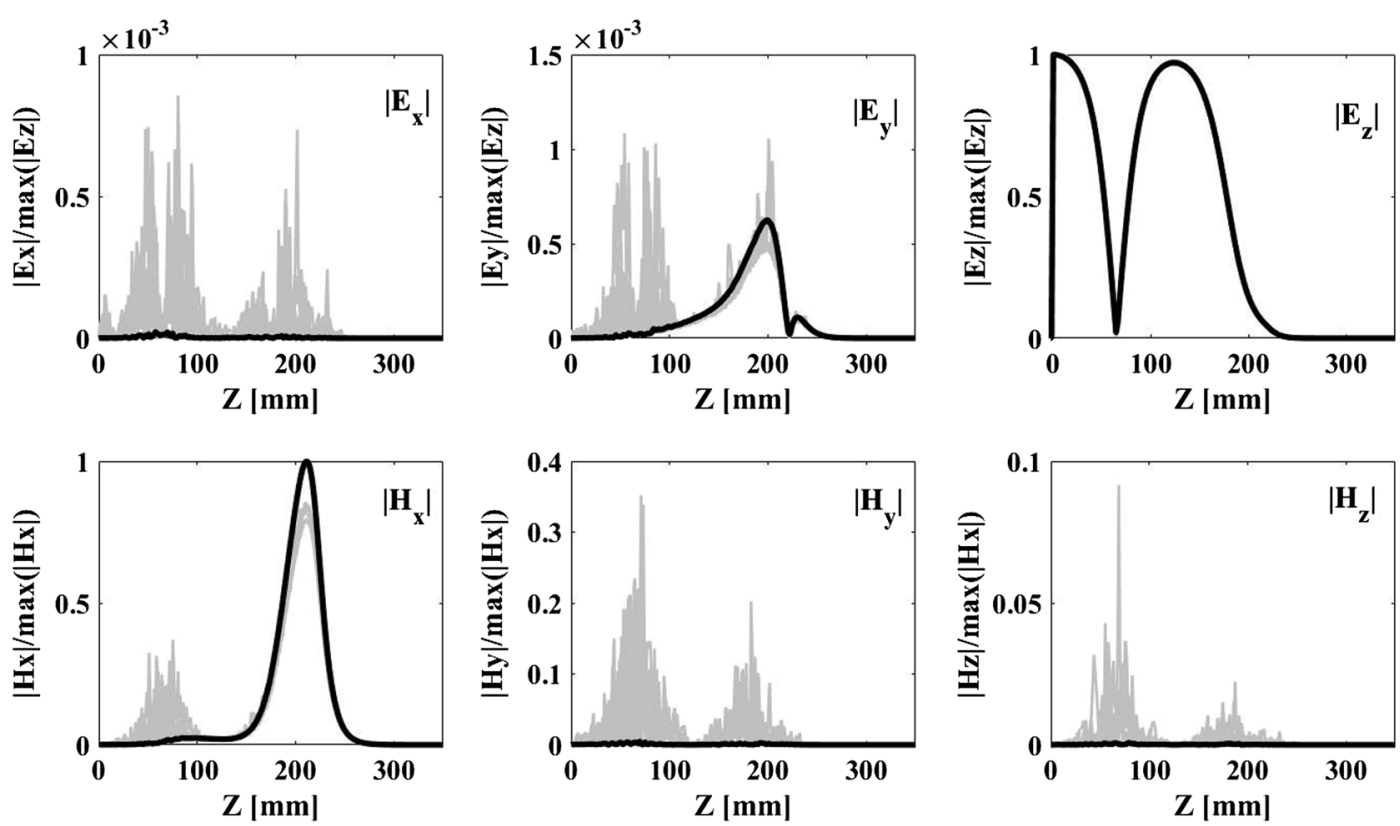

FIG. 3. Field components on the cavity axis. Black curves: converged results; Grey curves: history of intermediate results by refining mesh resolution of the simulation.

To enable excitation, a standard waveguide (WG) port condition is applied at the boundary of the input WG (see Fig. 2). The opposite side of the waveguide rf-feed port is closed. Based on a so-called monofrequency excitation method, two principal matching conditions (i.e., broadband matching from WG to coaxial line and narrowband matching from coaxial line to cavity) are satisfied by slightly tuning the length of the inner conductor. This results in a minimized reflection coefficient at the WG port position. The rf field is then calculated under such optimized conditions of the gun at its resonance frequency. Surface losses are taken into account. A 3D rf field map is extracted for use in particle tracking simulations $[8,39]$.

Figure 3 shows the computed normalized field distributions of the disturbed fundamental accelerating mode. The computational mesh resolution is refined to ensure numerical convergence. A local symmetric mesh along the cavity axis is applied to exclude numerical effects. As shown, pronounced $H_{x}$ and $E_{y}$ components are observed by the end of the coaxial line. The strength of these components reach their peak values in the transition between the end of the coaxial line and the cavity. Electron bunches passing through this transition region experience a vertical kick resulting from the Lorentz-force.

\section{SIMULATION OF THE KICK}

The kick is characterized by scanning the rf start-phase of the gun (with respect to photocathode laser phase) in particle tracking simulations. The beam centroid is initially placed at the center of the cathode plane. It is tracked through the gun cavity till close vicinity of the door-knob transition. The whole calculation domain is covered by the obtained 3D rf field map from Sec. II.

Figure 4 shows the particle offset from the axis on the transverse plane and the kick strength, defined as the ratio between the particle transverse momentum change and the longitudinal momentum along the cavity axis. Figures 4(b) and (d) show a vertical kick originating from the transition of the full cell of the gun to the coaxial line (from $\sim 180$ to $\sim 220 \mathrm{~mm}$ in Fig. 2). As shown, the kick strength further depends on the start-phase of the gun. At the maximum mean momentum gain $(\mathrm{MMMG})$ phase $\left(0^{\circ}\right)$, this leads to a
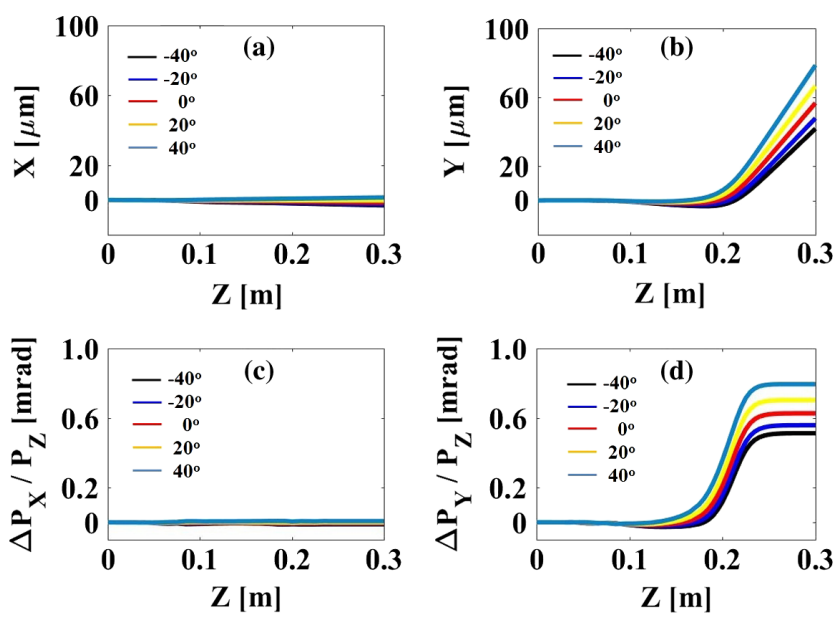

FIG. 4. Off-center distance of the beam centroid and the characterized kick strength for different rf start-phases. Note that $0^{\circ}$ in the legends corresponds to the gun phase for the maximum mean momentum gain (MMMG). 

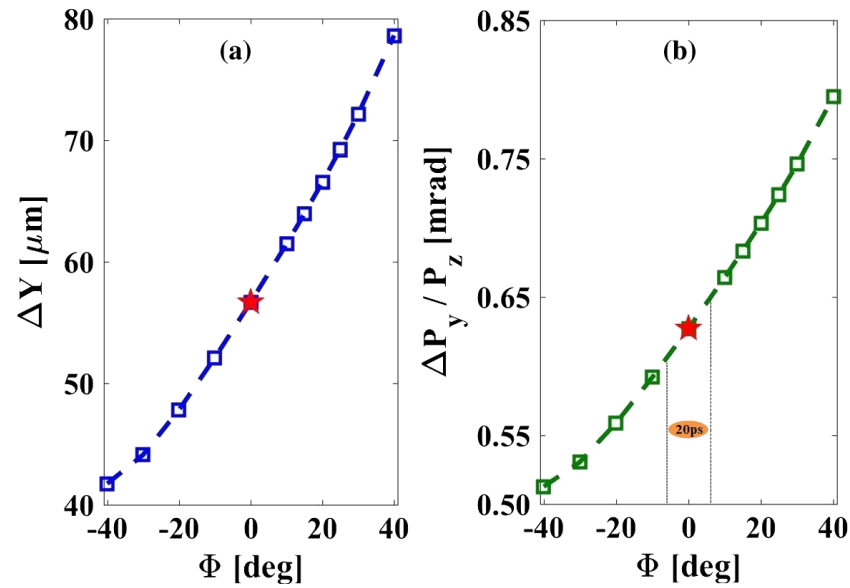

FIG. 5. Vertical displacement at $z=0.3 \mathrm{~m}$ and kick strength as a function of the gun phase. Note that 0 degree on both horizontal axes corresponds to the gun phase for the MMMG.

transverse kick of about 0.65 mrad [see Fig. 4(d)] for a maximum gun field gradient of about $60 \mathrm{MV} / \mathrm{m}$. A vertical offset of about $55 \mu \mathrm{m}$ is found at 0.3 meters downstream from the photocathode [Fig. 4(b)]. Note in addition, that there is almost no considerable kick in the horizontal direction for a large range of the $\mathrm{rf}$ phase with respect to the MMMG phase of the gun, as shown in Figs. 4(a) and $4(\mathrm{c})$

Figure 5 illustrates the kick strength and the particle offset from the axis as a function of the gun phase at $z=0.3 \mathrm{~m}$ where the kick strength is already stabilized [Fig. 4(d)]. As shown, the red markers denote the MMMG phase of the gun. Note that, a nominal electron bunch of 20 ps FWHM (full width half maximum) at PITZ corresponds to about 10 degrees gun phase at the resonant frequency of $1.3 \mathrm{GHz}$. Consequently, the head and tail of the electron bunch see a kick slope (see (b) in Fig. 5) due to the time dependency of the kick. This results in a kick difference of nearly $50 \mu \mathrm{rad}$ around the MMMG phase at a rf power level of $6.5 \mathrm{MW}$ in the gun. Given a further drift distance of about $5 \mathrm{~m}$ downstream the gun (where transverse emittance of the bunch is typically measured at PITZ), the resulting peak to peak orbit difference between electrons at bunch head and tail is roughly $250 \mu \mathrm{m}$. The bunch is thus tilted. A mismatch of the slices within the bunch may cause emittance growth. It should be also noted, that the presence of the imperfect solenoid fields [40] can further complicate the beam dynamics in a nonlinear manner. For simplicity, the gun solenoids are not included in these simulations.

\section{MULTIPOLE-EXPANSION-BASED KICK QUANTIFICATION}

To clarify the multipole composition and quantify their strengths in the characterized kick, the transverse momentum of the beam particle is presumably decomposed in a dipole component, a normal and a skew quadrupole component according to

$$
\Delta p_{x}(x, y, \phi)=\Delta p_{d x}+\left(k_{r f}+k_{\text {norm }}\right) x+k_{\text {skew }} y
$$

and

$$
\Delta p_{y}(x, y, \phi)=\Delta p_{d y}+\left(k_{r f}-k_{\text {norm }}\right) y+k_{\text {skew }} x .
$$

Here, $x, y, \Delta p_{x}$, and $\Delta p_{y}$ represent the particle offsets from the $x$ and $y$ axis at the integral kick location $(z \approx 0.2 \mathrm{~m})$ and the momentum changes, respectively. The term $\phi$ stands for the rf phase. The parameters $\Delta p_{d x}$ and $\Delta p_{d y}$ characterize the dipole kick while $k_{r f}, k_{\text {norm }}$, and $k_{\text {skew }}$ denote the $\mathrm{rf}$ focusing strength of the cylindrical symmetric mode, the normal and skew quadrupole kick, respectively. For simplicity, the gun solenoid is not considered in this approach.

Figure 6 shows an array of simulation particles initially placed on the cathode plane. These particles are tracked through the gun cavity passing by the end of the coaxial coupler till the door-knob transition at the MMMG phase of the gun. In Fig. 7, the particle transverse momentum change at $z=0.3 \mathrm{~m}$ is plotted as a function of the vertical and horizontal displacements at $z=0.18 \mathrm{~m}$ for all the particles. As shown, the transverse momentum in Figs. 7(a) and 7(d) is nearly linearly proportional to the displacement at the kick location. A vertical kick is observed in Fig. 7(b) while there is no kick on the horizontal plane. This is in good agreement with the findings in Fig. 4.

Based on the simulation results in Fig. 7, the multipoleexpansion forms Eqs. (1) and (2) are numerically fitted. This renders a pronounced vertical dipole kick $\left(\Delta p_{d y}\right)$ of $\sim 0.67 \mathrm{mrad}$. The horizontal dipole kick strength $\left(\Delta p_{d x}\right)$ is almost 0 . For the normal quadrupole component, the kick strength $k_{\text {norm }}$ is estimated as $\sim 1.5 \mu \mathrm{rad} / \mathrm{mm}$. The skew quadrupole component $k_{\text {skew }}$ is calibrated as $\sim 0.7 \mu \mathrm{rad} / \mathrm{mm}$.

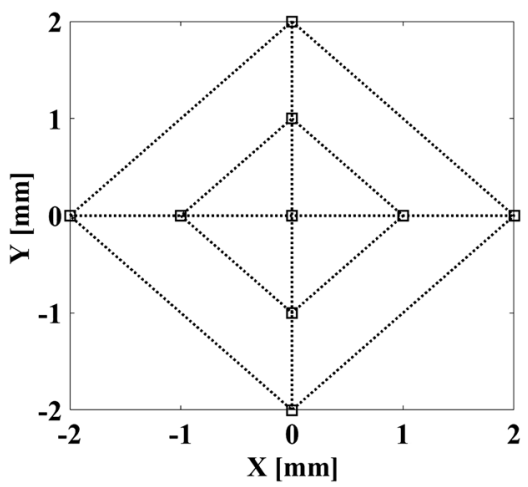

FIG. 6. Test particle positions on the cathode plane used in the simulations for kick quantification. 

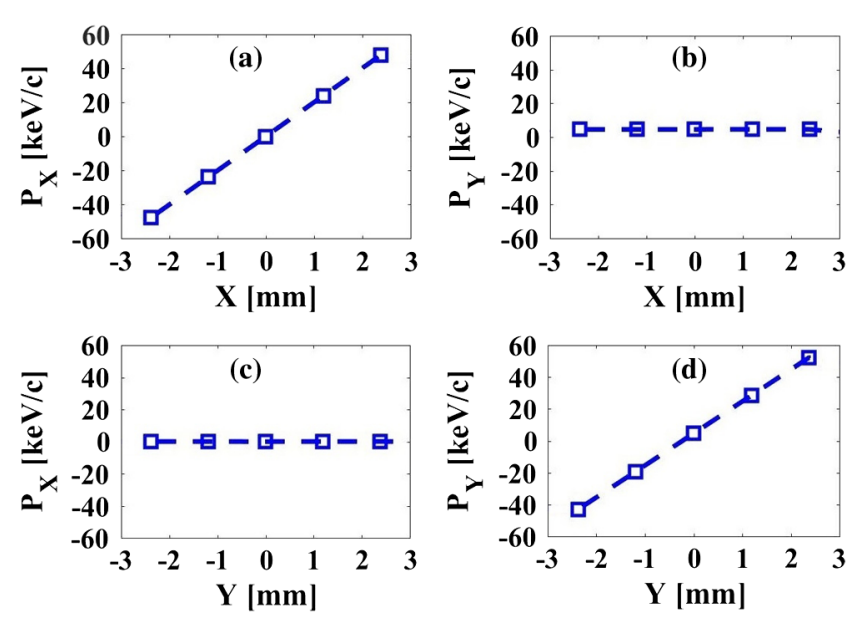

FIG. 7. Particle tracking simulation results for multipole expansion based quantification of the integral kick. The MMMG phase of the gun is used. The range of the axes are set the same in all subplots for illustration.

\section{FREQUENCY-DETUNING DEPENDENCY OF THE KICK}

\section{A. Frequency detuning}

A high rf input power of up to $6.5 \mathrm{MW}$ is required for the production of up to $650 \mu \mathrm{s}$ long trains of high-quality electron bunches. Such high rf power heats the surface of the standing-wave cavity and changes its resonance frequency. Due to thermal expansion, the resonant frequency of a cavity is linearly proportional to the temperature of the cavity. To reduce the temperature of the gun cavity, a water cooling scheme is applied at PITZ. To maintain gun operation at the resonance frequency, the gun temperature set-point is calculated as a function of the average rf power in the gun. The gun temperature is monitored using a temperature probe sitting close to the surface of the gun iris. This means, the change of the cooling water temperature for the gun changes the resonance frequency of the gun cavity.

For a quantitative characterization, the reflection coefficient $\left(S_{11}\right)$ is defined, which is the ratio of the reflected power over the forward power for a resonant cavity. The gun cavity has a resonance frequency $f_{0}(\sim 1.3 \mathrm{GHz}$ for the PITZ case) fed by the rf with a frequency $f$. In order to protect the microwave source (klystron), the reflected power from the cavity must be low (typically $\ll 2 \mathrm{MW}$ ). This requires a resonant frequency kept close to the $\mathrm{rf}$ frequency. The frequency detuning, $\Delta f=f-f_{0}$ is then used to describe how much the cavity is detuned from its resonance.

\section{B. Simulation}

To simulate the kick behavior in response to the frequency detuning, a simplification is made in the
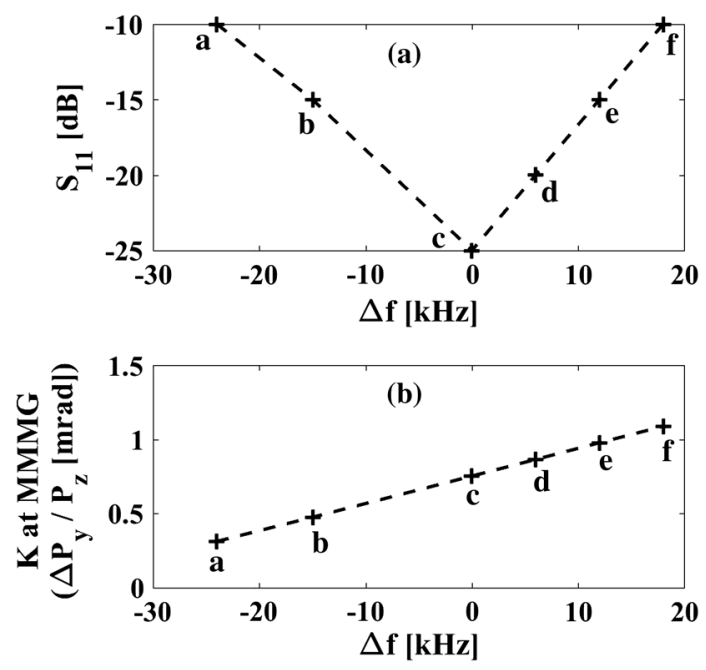

FIG. 8. Simulated reflection coefficient and kick dependency on frequency detuning.

simulations: the rf frequency is varied at the port position of the input waveguide (see Fig. 2) for defining the detuned frequency with respect to the cavity resonance. This allows computing 3D rf field distributions of the gun cavity at the detuned frequencies in frequency domain calculation. Using the obtained field distributions, particle tracking simulations using CST Particle Studio [8] are performed and the kick strength $(K$, the ratio of transverse beam momentum change over longitudinal momentum) can thus be computed at the detuned frequencies.

Figure 8(a) shows the simulated $S_{11}$ as a function of $\Delta f$ with points $a-f$ as the set-points for frequency detuning. Figure 8(b) illustrates the change of the kick strength over the detuned frequencies at the MMMG phase of the gun for the individual cases $a-f$. The beam momentum at the MMMG phase is kept the same $(\sim 6.8 \mathrm{MeV} / \mathrm{c})$ for all cases by adjusting the amplitudes of the applied rf field distributions in the simulations. As shown, the kick strength varies with the frequency detuning, and for the chosen set points, this shows a nearly linear behavior.

In Fig. 9, the phase dependency of the kick is shown for each case defined in Fig. 8 at the specified $\Delta f$, respectively. It is important to note, that the phase slope of the kick varies with the change of the frequency detuning. An overheating state $(\Delta f>0)$ of the gun cavity renders smaller phase slopes than in the overcooled cases $(\Delta f<0)$. More specifically, the effect leads to a flattened phase slope in the overheated case $(f)$ while resulting in the most significant slope (or curvature) for the overcooled case (a). Since a phase slope of the kick can result in the mismatch of slices within the bunch, a projected emittance growth can be expected. This also suggests, that operating the gun at its overheated state can effectively reduce (and even avoid) the emittance growth caused by the phasedependent kick for single bunch operation. It is also worthy 


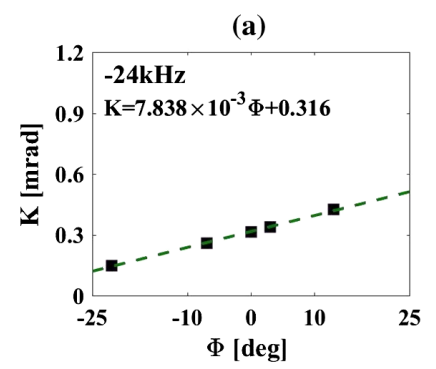

(c)

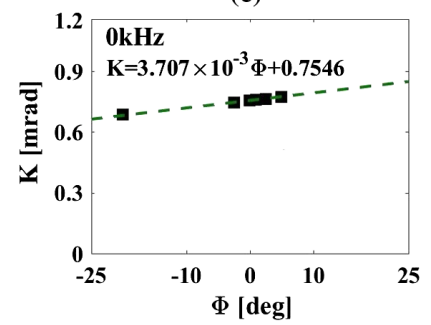

(e)
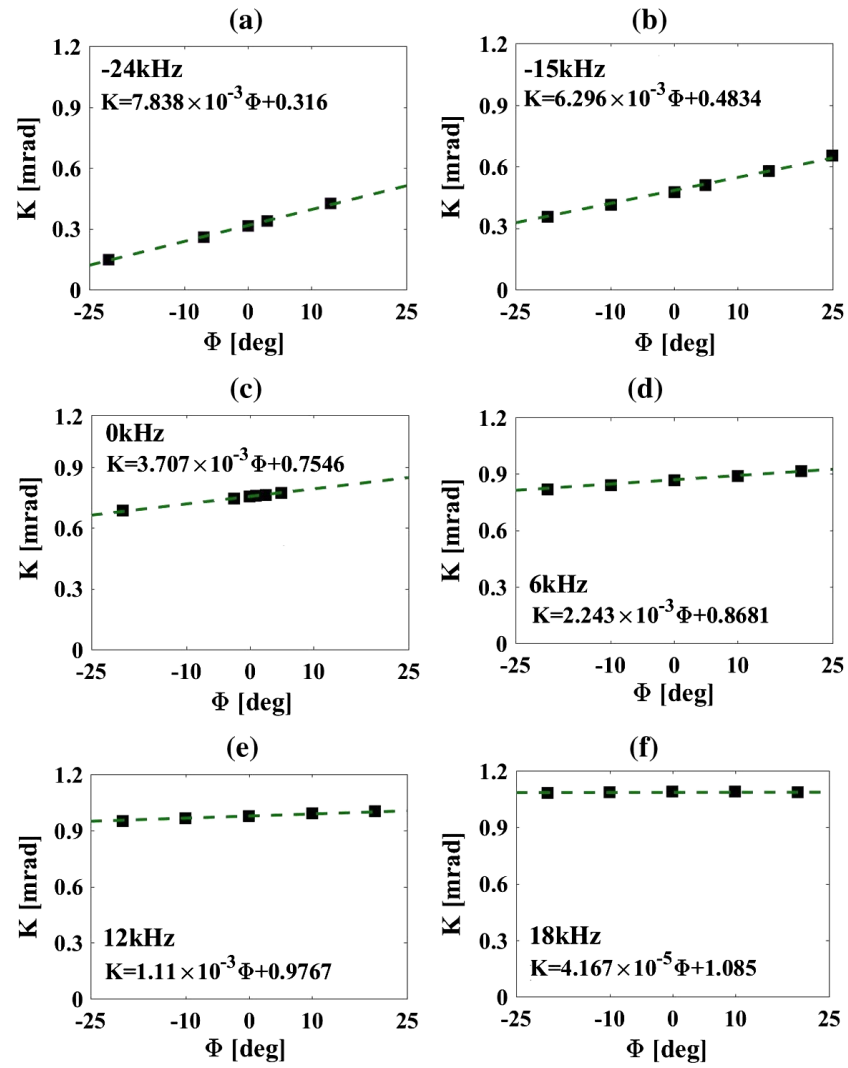

(d)

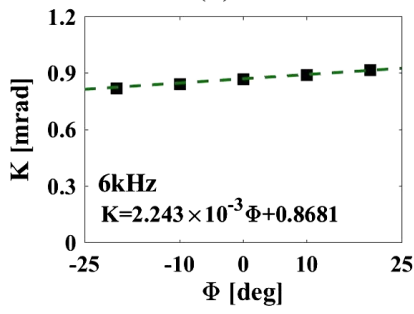

(f)

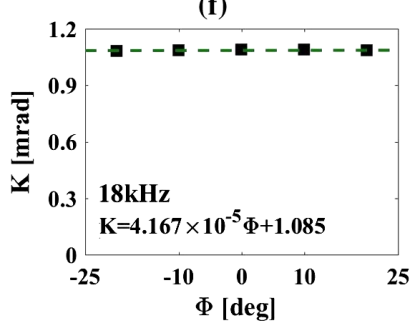

FIG. 9. Simulated phase slopes of the kick vs $\Delta f$. Green lines: linear fits to the simulation results. $\Phi$ : gun phase w.r.t. MMMG.

to note that the simulated behaviors, as shown in Fig. 9, coincide with the criterion for stable operation of the gun with high average power dissipation in routine experiments at PITZ.

\section{Experiment}

An experimental setup is sketched based on the PITZ beamline, as shown in Fig. 10. To measure the main dipole kick and characterize its dependencies on both rf gun phase and gun temperature, a single electron bunch is generated at the cathode $(z=0 \mathrm{~m})$ of the photo-gun and transported downstream till the observation screen $(z \approx 5.27 \mathrm{~m})$. The bunch position change in the radial direction is measured on the screen when changing the gun phase or the gun temperature. The latter is controlled by adjusting the cooling water temperature of the gun cavity. In the meanwhile this allows the adjustment of the frequency detuning (with respect to the timing of the electron bunch) within the rf pulse. For the measurements, the gun is adjusted to deliver a $\sim 6.28 \mathrm{MeV} / \mathrm{c}$ electron bunch at the gun exit when operated at its MMMG phase. For simplicity, the cut-diskstructure (CDS) booster is switched off. The gun solenoid current is fixed. Note in addition that for specifically characterizing the measurement set-point, the $S_{11}$ parameter (ratio between reflected and forward rf power) is derived from rf measurements.

Figure 11(a) shows the measured $S_{11}$ versus the cavity temperature with cases $\mathrm{A}, \mathrm{B}$, and $\mathrm{C}$ denoting the cooling states of the gun as overcooled, at resonance and overheated, respectively. For each case, the kick strength is measured in terms of the bunch position change $(\Delta r)$. This is conducted at a downstream observation screen $(\sim 5.27$ meters from cathode) as the gun phase is scanned. As shown, the phase slope of the kick is reduced as evolving from overcooling to overheating. Case $\mathrm{A}\left(\Delta \mathrm{r}_{\mathrm{p} 2 \mathrm{p}} \sim 0.9 \mathrm{~mm}\right)$ shows the strongest linear slope while case $\mathrm{C}\left(\Delta \mathrm{r}_{\mathrm{p} 2 \mathrm{p}} \sim\right.$ $0.4 \mathrm{~mm}$ ) delivers the smallest phase slope, particularly for the phase range of $M M M G \pm 5$ degrees $\left(\Delta \mathrm{r}_{\mathrm{p} 2 \mathrm{p}}<\right.$ $0.05 \mathrm{~mm}$ ). This is in fairly good agreement with the simulated kick behavior, as shown in Fig. 9. Figure 11(e) shows the measured variation of the radial bunch position as a function of the cavity temperature (i.e., frequency detuning of the cavity). The resonance temperature of the gun cavity is at $\sim 73.2^{\circ} \mathrm{C}$. Linear fits (green dashed lines) are given for the measurement data (black dots) in subplots (b)-(e), resembling the linear dependence found in simulations (Figs. 8 and 9).

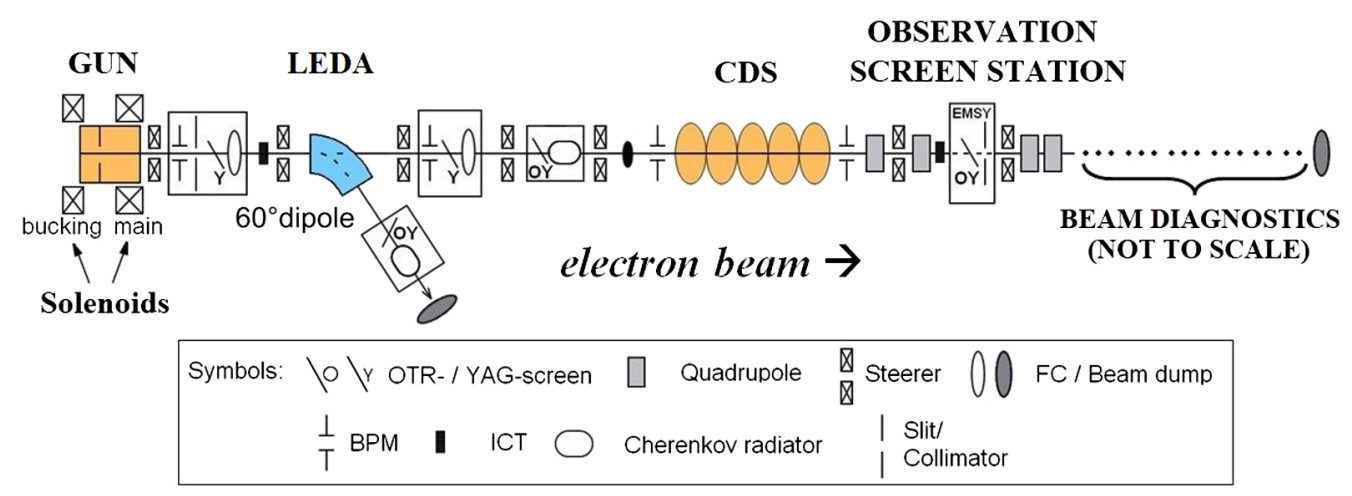

FIG. 10. Sketch of the partial PITZ beam line. CDS: cut-disk-structure. LEDA: low energy dispersive arm. 


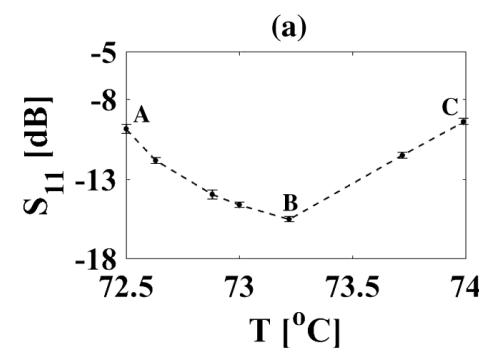

(c)

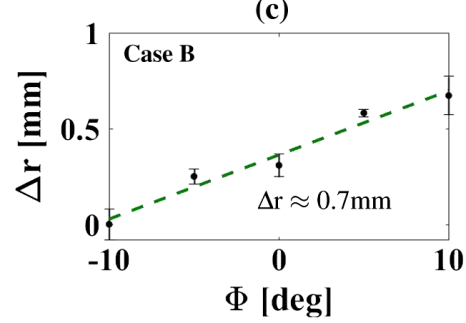

(b)

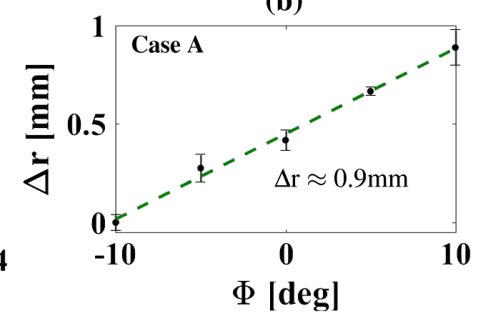

(d)

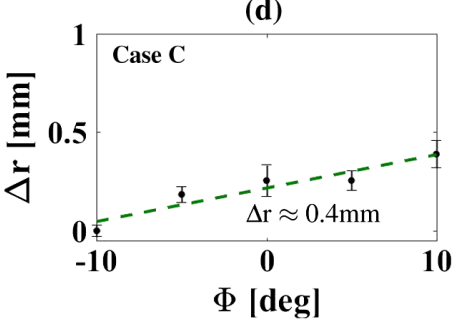

(e)

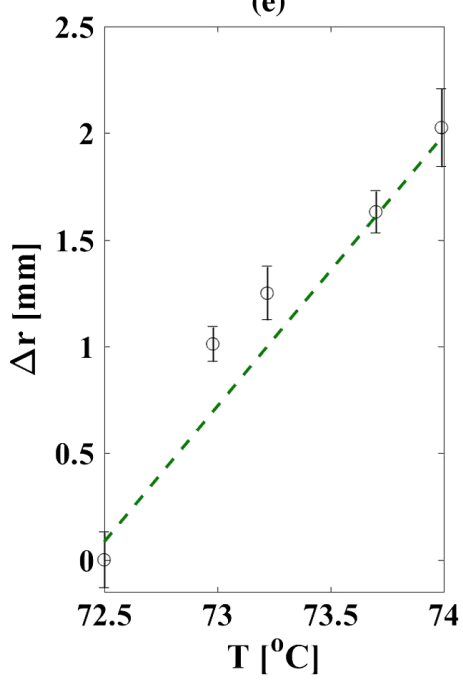

FIG. 11. Measured kick dependencies on gun phase and frequency detuning (or cavity temperature). Green lines: linear fits to the data. $\Phi$ : gun phase with respect to MMMG. The insets $\Delta$ r represent peak to peak changes.

\section{MEASUREMENT OF THE KICK IMPACTS ONTO BUNCH TRAIN OPERATION FOR FELS}

Figure 12 shows typical schematics for multiple correlations of the rf power reflection within the macro-pulse with gun cavity temperature, frequency detuning and timing of the electron bunch train within one rf pulse. As illustrated, frequency detuning of the gun cavity exists along the bunch train due to pulse rf heating. According to Sec. V, the identified kick varies as a function of the frequency detuning. Consequently, the bunches along the train see different kicks within the rf pulse. The effect is stronger for longer rf pulses as required for longer electron bunch trains. For the operation with long rf pulses of

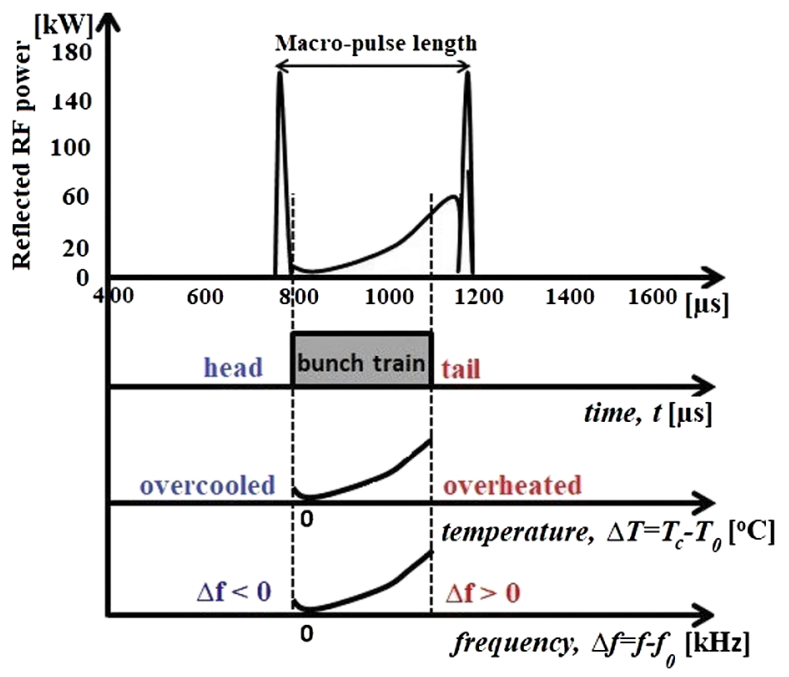

FIG. 12. Schematics of bunch train operation within rf macropulse with illustrative correlations with time, gun temperature change, and frequency detuning. several hundred microseconds, the impacts of the kick onto the properties of electron bunch trains are experimentally investigated. The position and size change of the bunches along the train are measured. This is done through measuring transverse properties of a single witness bunch moving along the long rf pulse by varying the cathode laser timing. The frequency detuning seen by the witness bunch and thus the frequency detuning dependent kick strength can be varied and measured. Note that the bunch charge and momentum are kept the same for all measurements.

\section{A. Orbit change}

Figure 13 shows the bunch position change along a $240 \mu \mathrm{s}$ train for a fixed beam momentum of $\sim 6.35 \mathrm{MeV} / \mathrm{c}$ at the MMMG phase of the gun. A peak to peak (P2P) position change of $\sim 650 \mu \mathrm{m}$ is obtained, which corresponds to roughly a P2P kick difference of $0.13 \mathrm{mrad}$ between the head and tail of the bunch train. Experimentally operating such a bunch train within a $300 \mu$ s long rf pulse results in roughly a $6 \mathrm{~dB}$ difference in the measured $S_{11}$ between the

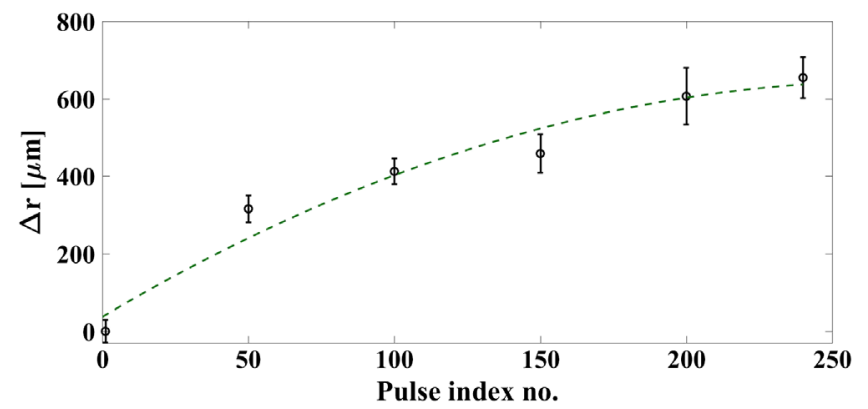

FIG. 13. Measured bunch position change along a $240 \mu$ s train at $\sim 5.27$ meters downstream from cathode. The solenoid current is fixed. Green curve: a polynomial fit to the data. 
head and tail of the train. This is in correspondence to the simulation results as shown in Fig. 8(a) for emulating the bunch train operation in between case $\mathrm{c}$ and case $\mathrm{d}$ in the overheated state of the gun. As shown in Fig. 8(b), the difference in the simulated kick strength along the train is estimated as $0.11 \mathrm{mrad}$, which is in fairly good agreement with the experimental findings although the acceleration gun gradient used in the simulation slightly differs from the measurement.

\section{B. Focusing change}

Furthermore, an RMS size change of the bunches along a train has been measured. Similar to measuring the orbit change in Sec. VI A, a witness bunch of about $20 \mathrm{pC}$ is moved along a long rf pulse (typically a few hundreds of microseconds) used for operating the bunch train (see Fig. 12). This is realized by changing the timing of the cathode drive laser with respect to the rf pulse. Through measuring the size of the witness bunch downstream on the observation screen $(z \approx 5.27 \mathrm{~m})$ at each scanning location along the rf pulse, the size of individual bunches along the train is measured. The impact of rf pulse heating onto the bunch train can thus be demonstrated in terms of the RMS bunch size change. Note that every time changing the cathode drive laser timing, the beam momentum is measured correspondingly at the low energy dispersive arm (LEDA, see Fig. 10). The intrabunch train momentum difference is monitored. Since the bunch size change is equivalent to the change of solenoid focusing in the injector, the effect is also evaluated by means of solenoid focusing change which is more relevant for the SASE tuning at FEL facilities. The focusing change is measured in terms of the difference in gun solenoid current along the train. The measurement is conducted for both the overfocusing and underfocusing case when the gun solenoid current is set to $355 \mathrm{~A}$ and $310 \mathrm{~A}$, respectively. As shown in Fig. 14(a) and 14(c), an RMS beam size change of $\sim 90 \mu \mathrm{m}$ is obtained between the head and tail of the bunch train for the overfocusing case while $\sim 65 \mu \mathrm{m}$ is measured for the case of underfocusing. In Fig. 14(b), a solenoid scan around

(a)

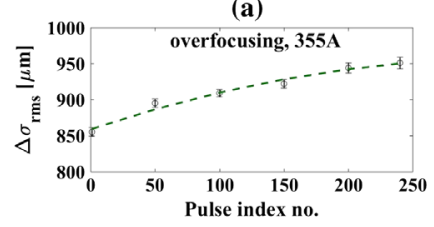

(c)

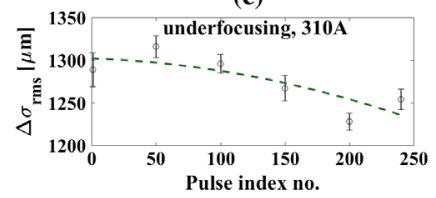

FIG. 14. Measured RMS bunch size and size change along a $240 \mu \mathrm{s}$ train for both the over- and underfocusing case.
TABLE I. Measured bunch momentum along a $240 \mu$ s train.

\begin{tabular}{lcc}
\hline \hline Bunch Index $^{\mathrm{a}}[$ number $]$ & Momentum $^{\mathrm{b}}[\mathrm{MeV} / \mathrm{c}]$ & $\left|\Delta \mathrm{P}_{z}\right|^{\mathrm{c}}$ \\
\hline 1 & $6.353 \pm 0.001$ & $5.0 \times 10^{-4}$ \\
50 & $6.346 \pm 0.003$ & $6.0 \times 10^{-4}$ \\
100 & $6.352 \pm 0.001$ & $3.5 \times 10^{-4}$ \\
150 & $6.349 \pm 0.004$ & $1.3 \times 10^{-4}$ \\
200 & $6.350 \pm 0.002$ & $3.1 \times 10^{-5}$ \\
240 & $6.349 \pm 0.003$ & $1.3 \times 10^{-4}$ \\
\hline \hline
\end{tabular}

${ }^{\mathrm{a}}$ Intratrain bunch sequential number.

${ }^{\mathrm{b}}$ Mean momentum $\approx 6.350 \mathrm{MeV} / \mathrm{c}$.

${ }^{\mathrm{c}}$ Momentum deviation with respect to mean momentum along the train.

355 A leads to a change of $\sim 33.8 \mu \mathrm{m}$ per ampere (overfocusing case). Likewise, $\sim 28.8 \mu \mathrm{m}$ per ampere is given for the underfocusing case [Fig. 14(d)]. This defines roughly a 2.7 A focusing change along the $240 \mu$ s bunch train for the overfocusing case and a 2.3 A change for the case of underfocusing. These effects should be more severe for longer rf pulses. The change of the orbit and size degrades the quality of the bunch train. For an FEL operated in such a long-pulse mode, this may disturb the SASE output power along a bunch train.

In addition, Table I shows the measured bunch momentum at LEDA at each scanned location (i.e., bunch index) along the rf pulse for a $240 \mu$ s bunch train when changing the cathode drive laser timing. As shown, the measured momentum change with respect to the mean momentum along the train is on the order of $10^{-4}$. This is one order of magnitude smaller than the measured focusing change due to the frequency detuning dependent coupler kick within the long rf pulse and is thus excluded as the cause of the observed intrabunch-train beam size variation.

\section{CONCLUSION}

A frequency-detuning dependent transverse rf coupler kick is analyzed in both simulation and experiment. A dedicated simulation and quantification approach suggests the composition of the kick as a main dipole component ( $~ 0.6 \mathrm{mrad}$ at $6.5 \mathrm{MW}$ ) and small normal and skew quadruple components. This agrees with the findings obtained in 3D simulations of the local rf field asymmetries. Furthermore, it is found that the kick depends on the start phase and the frequency detuning of the gun cavity. These transient behaviors of the kick are consistent between simulation and measurement. Due to the frequency detuning within the rf pulse, the resulting orbit and size change along the electron bunch train are exemplarily quantified for a $240 \mu \mathrm{s}$ train along a $300 \mu \mathrm{s}$ rf pulse. The beam momentum is kept at $\sim 6.35 \mathrm{MeV} / \mathrm{c}$, which corresponds to gun operation at $\sim 5.7 \mathrm{MW}$ at the MMMG phase. A P2P difference in the kick strength between the head and tail of the bunch train is estimated as $0.13 \mathrm{mrad}$, which is 
consistent with the simulation result. A P2P focusing change along the same train, in terms of the gun solenoid current, is quantified as $2.7 \mathrm{~A}$ and $\sim 2.3 \mathrm{~A}$ for the case of overfocusing and underfocusing, respectively. Schemes for the correction and compensation of the kick effects onto the bunch train operation are currently under discussion.

\section{ACKNOWLEDGMENTS}

The authors would like to thank F. Brinker, W. Decking, M. Dohlus, K. Floettmann, I. Zagorodnov, H. Schlarb from DESY for very useful discussions and comments on this subject. This work is supported by Deutsches ElektronenSynchrotron DESY.

[1] E. P. Team, The European X-Ray Free-Electron Laser Technical design report, Report No. DESY 2006-097, 2007.

[2] W. Decking and H. Weise, Commissioning of the european XFEL accelerator, in Proceeding of International Particle Accelerator Conference (IPAC'17), Copenhagen, Denmark, May, 2017, International Particle Accelerator Conference No. 8 (JACoW, Geneva, Switzerland, 2017), pp. 1-6, https://doi.org/10.18429/JACoW-IPAC2017MOXAA1.

[3] M. Altarelli et al., XFEL: The European X-Ray FreeElectron Laser: Technical design report, Report No. DESY 2006-097, DESY, 2006.

[4] W. Ackermann et al., Operation of a free-electron laser from the extreme ultraviolet to the water window, Nat. Photonics 1, 336 (2007).

[5] F. Stephan et al., Detailed characterization of electron sources yielding first demonstration of European X-ray Free-Electron Laser beam quality, Phys. Rev. Accel. Beams 13, 020704 (2010).

[6] M. Krasilnikov et al., Experimentally minimized beam emittance from an L-band photoinjector, Phys. Rev. Accel. Beams 15, 100701 (2012).

[7] Y. Renier et al., Statistics on high average power operation and results from the electron beam characterization at PITZ, in Proceeding of International Particle Accelerator Conference (IPAC'17), Copenhagen, Denmark, 2017, International Particle Accelerator Conference No. 8 (JACoW, Geneva, Switzerland, 2017), pp. 1806-1808, https:// doi.org/10.18429/JACoW-IPAC2017-TUPIK051.

[8] Computer Simulation Technology CST, http://www.cst.de (2018).

[9] R. E. Collin, Foundations for Microwave Engineering (McGraw-Hill, New York, 1992).

[10] D. Alesini, Power coupling, arXiv:1112.3201.

[11] B. Dwersteg, K. Floettmann, J. Sekutowicz, and C. Stolzenburg, RF gun design for the TESLA VUV Free Electron Laser, Nucl. Instrum. Methods Phys. Res., Sect. A 393, 93 (1997), free Electron Lasers 1996.

[12] A. Oppelt et al., Tuning, conditioning, and dark current measurements of a new gun cavity at PITZ, in Proceeding of International Free Electron Laser Conference (FEL'06), Berlin, Germany, 2006, International Free
Electron Laser Conference No. 28 (JACoW, Geneva, Switzerland, 2006), pp. 609-612, http://accelconf.web .cern.ch/AccelConf/f06/PAPERS/THPPH023.PDF.

[13] C. Limborg, Z. Li, L. Xiao, J. F. Schmerge, D. Dowell, S. Gierman, E. Bong, and S. Gilevich, RF design of the LCLS gun, Report No. LCLS-TN-05-3, 2005.

[14] J. Schmerge, A. Brachmann, D. Dowell, A. Fry et al., The LCLS-II injector design, in Proceeding of Free-Electron Laser Conference (FEL'14), Basel, Switzerland (JACoW Publishing, Geneva, Switzerland, 2015), pp. 815-819, http://accelconf.web.cern.ch/AccelConf/FEL2014/papers/ thp042.pdf.

[15] R. A. Marsh, F. Albert, S. G. Anderson, G. Beer, T. S. Chu, R. R. Cross et al., Modeling and design of an X -band rf photoinjector, Phys. Rev. Accel. Beams 15, 102001 (2012).

[16] H. J. Qian, H. B. Chen, Y. C. Du, W. H. Huang, C. Li et al., Design and cold tests of a prototype photocathode $\mathrm{rf}$ gun for shanghai sxfel facility, in Proceeding of International Particle Accelerator Conference (IPAC'11), San Sebastian, Spain (EPS-AG, Spain, 2011), pp. 31703172, http://accelconf.web.cern.ch/AccelConf/IPAC2011/ papers/thpc121.pdf.

[17] D. Alesini, A. Gallo, B. Spataro, A. Marinelli, and L. Palumbo, Design of couplers for traveling wave RF structures using 3D electromagnetic codes in the frequency domain, Nucl. Instrum. Methods Phys. Res., Sect. A 580, 1176 (2007).

[18] N. M. Kroll et al., Applications of time domain simulation to coupler design for periodic structures, Monterey, CA, USA Report No. SLAC-R-561, 2000.

[19] S. Zheng et al., A quantitative method of coupler cavity tuning and simulation, in Proceeding of the Particle Accelerator Conference (PAC'O1), Chicago, IL, USA (IEEE, Piscataway, NJ, 2001), pp. 981-983, http://accelconf.web .cern.ch/AccelConf/p01/PAPERS/MPPH091.PDF.

[20] L. Xiao, R. Boyce, D. Dowell, Z. Li, C. Limborg-Deprey, and J. F. Schmerge, Dual feed rf gun design for the lcls, in Proceeding of the Particle Accelerator Conference, Knoxville, Tennessee (IEEE, Piscataway, NJ, 2005), pp. 3432-3434, http://accelconf.web.cern.ch/AccelConf/ p05/PAPERS/TPPE058.PDF.

[21] D. H. Dowell, E. Jongewaard, J. Lewandowski, C. Limborg-Deprey, Z. Li, J. Schmerge et al., The development of the linac coherent light source rf gun, ICFA Beam Dynamics Newsletter 46, 162 (2008).

[22] LCLS-II Project Team, LCLS-II Final Design Report DRAFT, 2014.

[23] M. S. Chae, J. H. Hong, Y. Parc, I. S. Ko, S. J. Park, H. J. Qian, W. H. Huang, and C.X. Tang, Phys. Rev. Accel. Beams 14, 104203 (2011).

[24] X. Guan, C. Tang, H. Chen, W. Huang, X. He, P. Xu, and R. Li, Study of RF-asymmetry in photo-injector, Nucl. Instrum. Methods Phys. Res., Sect. A 574, 17 (2007).

[25] B. Buckley and G. H. Hoffstaetter, Transverse emittance dilution due to coupler kicks in linear accelerators, Phys. Rev. Accel. Beams 10, 111002 (2007).

[26] D. H. Dowell, F. Zhou, and J. Schmerge, Exact cancellation of emittance growth due to coupled transverse dynamics in 
solenoids and rf couplers, Phys. Rev. Accel. Beams 21, 010101 (2018).

[27] M. Dohlus et al., The influence of the main-coupler field on the transverse emittance of a superconducting rf gun, in Proceeding of European Particle Accelerator Conference (EPAC'04), Lucerne, Switzerland (EPS-AG, Lucerne, 2004), http://accelconf.web.cern.ch/AccelConf/e04/ PAPERS/MOPKF013.PDF.

[28] K. J. Kim, Rf and space-charge effects in laser-driven rf electron guns, Nucl. Instrum. Methods Phys. Res., Sect. A 275, 201 (1989).

[29] M. Krasilnikov et al., Impact of the rf-gun power coupler on beam dynamics, in Proceeding of European Particle Accelerator Conference, (EPAC'02), Paris, France, 2002, European Particle Accelerator Conference No. 8 (JACoW, Geneva, Switzerland, 2002), pp. 1640-1642, http:// accelconf.web.cern.ch/AccelConf/e02/PAPERS/ WEPRI077.pdf.

[30] R. Cee, M. Krassilnikov, S. Setzer, and T. Weiland, Beam dynamics simulations for the pitz $\mathrm{rf}$ gun, in Proceeding of European Particle Accelerator Conference, (EPAC'O2), Paris, France (EPS-IGA and CERN, Geneva, 2002), pp. 1622-1624, http://accelconf.web.cern.ch/AccelConf/ e02/PAPERS/WEPRI079.pdf.

[31] F. Sannibale, D. Filippetto, M. Jhnson, D. Li, T. Luo, C. Mitchell, J. Staples, S. Virostek, R. Wells, and J. M. Byrd, Upgrade possibilities for continuous wave rf electron guns based on room-temperature very high frequency technology, Phys. Rev. Accel. Beams 20, 113402 (2017).

[32] F. Sannibale, D. Filippetto, H. Qian, C. Mitchell, F. Zhou, T. Vecchione, R. K. Li, S. Gierman, and J. Schmerge, High-brightness beam tests of the very high frequency gun at the Advanced Photo-injector experiment test facility at the Lawrence Berkeley National Laboratory, Rev. Sci. Instrum. 90, 033304 (2019).

[33] D. Li, H. Feng, D. Filippetto, M. Johnson, A. Lambert, T. Luo et al., Recent progress on the design of normal conducting APEX-II VHF CW electron gun, in Proceeding of International Particle Accelerator Conference (IPAC'19), Melbourne, Australia (JACoW Publishing, Geneva, Switzerland, 2019) pp. 1891-1894, https:// doi.org/10.18429/JACoW-IPAC2019-TUPRB097.
[34] F. Sannibale, B. Bailey, K. Baptiste, A. Catalano, D. Colomb, J. Corlett et al., Status of the lbnl normalconducting $\mathrm{cw}$ vhf electron photo-gun, in Proceedings of the 32nd Free Electron Laser Conference, Malmöö, Sweden (Max-lab, Sweden, 2010), pp. 475-478, http://accelconf.web.cern.ch/AccelConf/FEL2010/papers/ wepb36.pdf.

[35] Z.-T. Zhao et al., in Proceeding of International FreeElectron Laser Conference (FEL'11), Shanghai, China (JACoW Publishing, Geneva, Switzerland, 2011).

[36] G. Wu, H. Wang, C. E. Reece, and R. A. Rimmer, Waveguide coupler kick to beam bunch and current dependency on srf cavities, in Proceeding of the 13th International Workshop on RF Superconductivity (SRF'07), Beijing, China, October, 2007 (JACoW, Geneva, Switzerland, 2007), pp. 721-723, http://accelconf.web.cern.ch/ AccelConf/srf2007/PAPERS/WEP85.pdf.

[37] Y. Chen et al., Coaxial coupler RF Kick in the PITZ rf gun, in Proceeding of International Free Electron Laser Conference (FEL'17), Santa Fe, NM, USA, August 20-25, 2017, International Free Electron Laser Conference No. 38 (JACoW, Geneva, Switzerland, 2018), pp. 422-425, https://doi.org/10.18429/JACoWFEL2017-WEP005.

[38] Y. Chen, H. Qian, M. Krasilnikov, F. Brinker, W. Decking, J. Good, M. Gross, P. Huang, I. Isaev, C. Koschitzki, S. Lal, X. Li, O. Lishilin, G. Loisch, D. Melkumyan, R. Niemczyk, A. Oppelt, H. Shaker, G. Shu, F. Stephan, and G. Vashchenko, Frequency-detuning dependent transient coaxial rf coupler kick, in Proceeding of International Free-Electron Laser Conference (FEL'19), Hamburg, Germany (JACoW Publishing, Geneva, Switzerland, 2019), https://doi.org/ 10.18429/JACoW-FEL2019-THP007.

[39] K. Floettmann, ASTRA particle tracking code, http://www .desy.de/ mpyflo/ (2017).

[40] M. Krasilnikov et al., Electron beam asymmetry compensation with gun quadrupoles at PITZ, in Proceeding of International Free Electron Laser Conference (FEL'17), Santa Fe, NM, USA, August 20-25, 2017, International Free Electron Laser Conference No. 38 (JACoW, Geneva, Switzerland, 2018), pp. 429-431, https://doi.org/10.18429/ JACoW-FEL2017-WEP007. 